\title{
Local ablative therapy of brain metastasis from non-small cell lung cancer: benefits and limitations
}

\author{
Felipe de Azevedo Rosas, Sérgio Leonardo Favareto, Douglas Guedes de Castro \\ Department of Radiation Oncology, AC Camargo Cancer Center, São Paulo, Brazil \\ Contributions: (I) Conception and design: All authors; (II) Administrative support: All authors; (III) Provision of study materials or patients: All \\ authors; (IV) Collection and assembly of data: All authors; (V) Data analysis and interpretation: All authors; (VI) Manuscript writing: All authors; (VII) \\ Final approval of manuscript: All authors. \\ Correspondence to: Douglas Guedes de Castro, MD, MSc, PhD. Department of Radiation Oncology, AC Camargo Cancer Center, São Paulo, Brazil. \\ Email: dougguedes@uol.com.br.
}

\begin{abstract}
Brain metastases (BMs) are the most common intracranial tumors and non-small cell lung cancer (NSCLC) are responsible for BM more than any other solid tumor. Its frequency is increasing due to of the availability of new imaging techniques, earlier diagnosis and improvement in treatment techniques and survival rates. NSCLC patients with BM represent heterogeneous prognostic group. The possibility of better prognostic stratification associated with more systemic therapy options and imaging and radiation technology advances have led to an increment of evaluation and indication of local ablative radiotherapy. The definite increment in quality of life and the potential overall survival (OS) benefit of its indication must be balanced with eventual higher risk of brain disseminated disease when whole brain irradiation is postponed. Therefore, a multidisciplinary evaluation is recommended to refine and personalize the therapeutic approach. The development of clinical nomograms or evaluation of circulating tumor cells/tumoral DNA that predict the survival free of new lesions may be the tools that will warranty further optimization of the treatment of NSCLC patients with BM. In this review, we report the main aspects of diagnosis, prognosis and therapeutic options and dilemmas evolving local ablative radiotherapy essentially based on seminal, updated prospective studies and ongoing trials.
\end{abstract}

Keywords: Brain metastasis (BM); non-small cell lung cancer (NSCLC); radiosurgery; stereotactic radiotherapy (SRT)

Submitted Feb 21, 2020. Accepted for publication Oct 12, 2020.

doi: $10.21037 /$ jtd-19-3321

View this article at: http://dx.doi.org/10.21037/jtd-19-3321

\section{Introduction}

In 2018, lung cancer was the third leading cause of cancer $(470,000,12 \%$ of total $)$ and the leading cause of cancer death, with an estimated of 388,000 deaths $(20 \%)$ in Europe. Non-small cell lung cancer (NSCLC) represents approximately $85 \%$ of all cases of lung cancer $(1,2)$.

Brain metastases (BMs) are the most common intracranial tumors and NSCLC is responsible for BM more than any other solid tumor, representing $43 \%$ of all cancers (3). It is estimated that $20 \%$ of patients with NSCLC have BM at diagnosis and $30-50 \%$ will be diagnosed during follow-up (4).
Its frequency is increasing due to the availability of new imaging techniques, earlier diagnosis and improvement in treatment techniques and survival rates (5).

\section{Diagnosis}

Magnetic resonance imaging (MRI) with intravenous contrast is preferable than computed tomography (CT) for the detection of BM (6). While CT can make a firstline approach and give fast information on intracranial hemorrhage, herniation, mass effect, and hydrocephalus, it has low resolution and low sensitivity for differential 


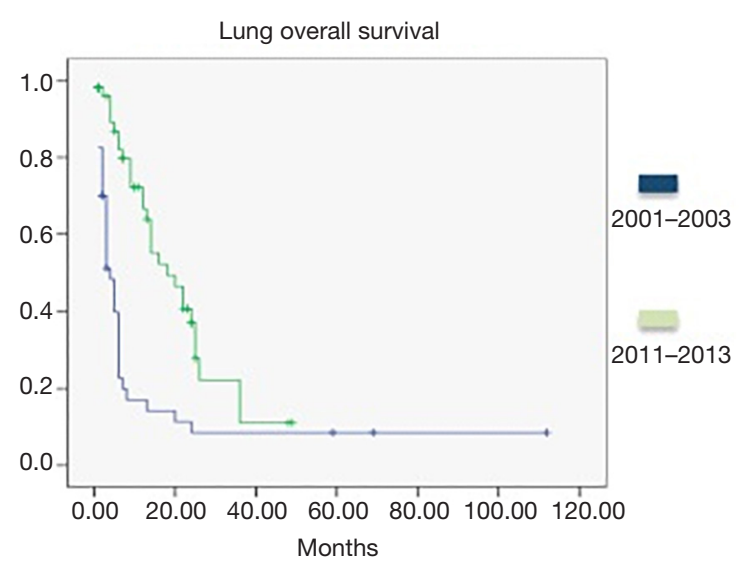

Figure 1 Kaplan-Meier curve for OS by period of treatment of $\mathrm{BM}$ of lung cancer with 40 patients in the initial cohort and 51 patients in the more recent cohort. OS, overall survival; BM, brain metastasis.

diagnosis. MRI has greater sensitivity than CT, with major resolution and better lesion characterization and localization (7).

A peripheral location, spherical shape, ring enhancement with extensive peritumoral edema and multiple lesions suggest a metastatic disease on the MRI, but there are no pathognomonic signs that define this diagnosis (8). Differential diagnosis, such as primary brain tumors and non-neoplastic conditions, are difficult to differentiate from BM. With the development of MRI techniques, such as MR proton spectroscopy (MRS), MR perfusion-including fluid attenuated inversion recovery (FLAIR), diffusion-weighted imaging (DWI), and diffusion tensor imaging (DTI) the detection, treatment planning and follow-up of this condition has become easier (9).

\section{Prognosis}

On historical data, the prognosis of BM has been estimated in 2-6 months, but it has changed over the years with improved options for local and systemic therapy (10). Many models of survival prediction after diagnosis are used, initially with the recursive partitioning analysis (RPA). Subsequently, the Diagnosis-Specific Graded Prognostic Assessment (DS-GPA) was developed to overcome some limitations that RPA had and it was updated through time (11).

At AC Camargo Cancer Center, we observed a shifting landscape of overall survival (OS) in patients with BM in a 10 -year timeframe based on a database analysis of consecutive patients with $\mathrm{BM}$ from melanoma, breast and NSCLC treated between 2001 to 2003 and 2011 to 2013. Among the 91 patients with NSCLC, the median OS was 4 months for 40 patients in the initial cohort and 18 months for 51 patients the recent cohort $\left(\mathrm{P}_{\text {log-rank }}<0.0001\right.$; HR: 2.79 , 95\% CI: 1.68-6.32, $\mathrm{P}<0.0001$ ) (Figure 1). It is plausible that these differences are due to institutional factors, such as the implementation of stereotactic radiotherapy (SRT) techniques-fractionated stereotactic radiotherapy (SFRT) or single dose, stereotactic radiosurgery (SRS)—and/or changing practice patterns such as the increase of systemic therapy alternatives and more available options in diagnostic methods between the two cohorts. These findings reinforce that prognostic indexes should be constantly updated, in order to refine therapeutic decision (12).

The most recent update of the DS-GPA for lung cancer incorporates molecular markers into the algorithm (lungmolGPA), which shows that the presence of EGFR or ALK gene alterations is associated with delayed onset of BM and longer median OS compared to those without these alterations (13). A delay of local treatment with the use of systemic therapy, including antiangiogenic agents, targeted therapies and immune-checkpoint inhibitors, may be considered as the first-line therapy, with the availability of salvage radiotherapy for patients with asymptomatic BM.

\section{Whole brain radiation therapy (WBRT)}

Historically, this group of patients were treated with either WBRT or best supportive care with steroids. Later, the addition of a local approach improved outcomes when combined with WBRT, leading to lower brain recurrences, increased median survival and functional autonomy with surgery and improved functional autonomy and survival for patients with a single unresectable BM with SRS $(14,15)$.

WBRT is currently the standard of care for disseminated $\mathrm{BM}$, with a dose of 30 Gy in 10 fractions or short course of 20 Gy in 5 fractions (depending on the clinical conditions of the patients). In patients with BM of NSCLC, poor performance status and who were not candidates for SRS, the QUARTZ trial showed no significant difference in OS and quality of life between dexamethasone and better supportive care with or without WBRT (16).

\section{Stereotactic radiotherapy}

Recently, the use of SRT alone or combined with other treatments proved to be a safe and effective option, being 
now one of the cornerstones in the treatment of $\mathrm{BM}$ in NSCLC patients. However, each approach has its advantages and drawbacks, making it more or less suitable for each singular patient. Instead of a "one to fit them all" approach, optimal treatment strategy should be tailored to each situation based on tumor and patient information. Here we review the role of SRT in treating BM on NSCLC.

The first evidence supporting the benefits of adding SRS to the treatment of BM started to appear in the late 90's. Kondziolka et al. randomized 27 patients with 2-4 BM with a limit diameter of $25 \mathrm{~mm}$ to upfront WBRT (30 Gy in 12 fractions) or WBRT + SRS. The SRS boost greatly improved local control ( $92 \%$ vs. $0 \%$ in 1 year) with no neurologic or systemic morbidity (17). Later, the results of a large phase III trial conducted by the RTOG group supported this evidence. The RTOG 9508 enrolled 333 patients with 1-3 BM and randomly allocated to either WBRT or WBRT + SRS boost. Although there was no OS difference among the two groups, patients with single BM had a statistical significant improvement in OS (4.9 vs. 6.5 months) with the addition of SRS boost (15). Later on, in 2014, a secondary analysis of RTOG 9508 results stratified the lung cancer patients (211/333) by GPA score. This analysis found that the subset of patients with GPA 3.5-4.0 had improved OS with the SRS boost addition to their treatment $(21 \mathrm{vs}$. 10.3 months, $\mathrm{P}=0.05$ ) regardless of the number of $\mathrm{BM}$ even though the benefit was still greater for patients with unique BM (18).

These trials carved a place for SRS in the treatment strategy of NSCLC patients with BM. The next step was to assess if WBRT could be entirely replaced. Aoyama et al., in the Japanese Radiation Oncology Study Group (JROSG) 99-1, randomized 132 patients with 1-4 BM to receive either SRS alone or SRS + WBRT. There was no improvement in survival with the inclusion of WBRT, but patients receiving isolated SRS had more intracranial relapse than the subgroup that received WBRT (46.8\% vs. $76.4 \%$ intracranial relapse in 1 year) (19). Patients who received WBRT + SRS had higher probability of necrosis and leukoencephalopathy. Regarding cognition, no difference between the groups was established. However, patients were evaluated with the mini-mental status exam, which is suboptimal for neurocognition assessment. Even though this trial found no difference in cognitive impairment between the arms, it is well established in several other trials the cognitive toxicity of WBRT (20-24). Therefore, despite the better local control of WBRT + SRS, treatment with SRS alone provided better quality of life and patients with intracranial failures can be adequately salvaged with no impact on OS. A phase III trial conducted by the EORTC group assessed if adding WBRT to patients with 1-3 BM initially submitted to treatment with SRS or surgery could improve the duration of functional independence. The results corroborated Aoyama's findings regarding local control of SRS and failed to demonstrate longer functional independence with the addition of WBRT (25). These trials consolidated SRS as a main tool to be used upfront on the oligometastatic scenario.

No significant difference was found between surgical resection and SRS in NSCLC patients with single BM (26). Also, surgical resection with adjuvant radiotherapy-with SRS - can be used, with no difference in terms of OS when comparing to WBRT, with lower probability of decline in cognitive function with SRS (27).

Up until this point, for patients with more extensive brain disease ( $>4 \mathrm{BM}$ ), WBRT was preferred based on the assumption that these patients will have poor survival and will necessarily fail elsewhere in the brain. This belief is based on the rationale that $\mathrm{BM}$ results from hematogenous dissemination making it reasonable to assume that the whole brain may be seeded with micrometastatic disease. In 2014, Yamamoto et al. published the results of a large prospective trial designed to examine if SRS without WBRT in treating patients with 5-10 BM was non-inferior to that for patients with oligometastatic brain disease. This trial enrolled 1,194 patients, 208 of them with 5-10 BM. There was no difference in OS between patients treated with upfront SRS with 2-4 BM and patients with 5-10 BM (HR: 0.97, 95\% CI: 0.81-1.18, $\mathrm{P}=0.78 ; \mathrm{P}_{\text {non-inferiority }}<0.0001$ ) (28). There was also no difference in distant brain relapse or toxicity related to SRS between the groups. A recently published update of this trial also concluded that local tumor progression did not differ between the groups (29). Currently, there are two trials recruiting patients with multiple BM to further assess the SRS use in this scenario. One of them, conducted by the Dana Farber Cancer Institute, is a phase III trial of SRS vs. WBRT as upfront treatment for patients with 5-20 BM (30). Other is led by National Cancer Information Center, which is randomizing patients with 5-15 BM to receive either SRS or WBRT with memantine and hippocampal avoidance. Primary endpoints are OS and neurocognitive progressionfree survival (31).

In light of the evidence that patients with more than four BM may be treated with SRS, Lester-Coll et al. conducted a study to determine the cost-effectiveness of the 
three main therapeutic strategies involving radiotherapy: SRS, SRS + WBRT and WBRT in patients with up to $10 \mathrm{BM}$. The endpoints included cost, quality-adjusted life years (QALYs), and incremental cost-effectiveness ratios. They concluded that SRS alone is the most cost-effective treatment (32).

In spite of the current trend of using SRS alone, mainly for oligometastatic brain disease, there may be a role for WBRT for some lung cancer patients. A provocative finding of a secondary analysis of the study JROSG 99-1 restricted to NSCLC BM was a significant better OS in the more favorable DS-GPA (2.5-4) patients in WBRT + SRS vs. SRS alone, with a median survival time of 16.7 (95\% CI: 7.5-72.9) vs. 10.6 (95\% CI: 7.7-15.5) months $(\mathrm{P}=0.04)$ (33). This finding suggests that WBRT may have a role in the brain disease control and consequent OS of oligometastatic NSCLC patients, although the best timing is not defined. On the opposite way, a similar exploratory analysis of the North Central Cancer Treatment Group N0574 (Alliance) randomized controlled trial of SRS with or without WBRT for limited, 1-3 BM of NSCLC observed no significant difference in OS according to receipt of WBRT in favorable-prognosis patients, which would support the approach of SRS alone in the majority of patients with limited BM (34). These both studies suggest that a fine tuning between SRS and WBRT must be defined in order to optimize the control of brain disease.

\section{Conclusions}

NSCLC patients with BM represent heterogeneous prognostic group. The possibility of better prognostic stratification associated with more systemic therapy options and imaging and radiation technology advances have led to an increment of evaluation and indication of local ablative radiotherapy. The definite preservation in quality of life and the potential OS benefit of its indication must be balanced with eventual higher risk of brain disseminated disease when WBRT is postponed. The development of clinical nomograms or evaluation of circulating tumor cells/tumoral DNA that predict the survival free of new lesions may be the tools that will warranty further optimization of the treatment of NSCLC patients with BM.

\section{Acknowledgments}

Funding: None.

\section{Footnote}

Provenance and Peer Review: This article was commissioned by the Guest Editor (Lucyna Kepka) for the series "Radiotherapy for Brain Metastases from Lung Cancer" published in Fournal of Thoracic Disease. The article has undergone external peer review.

Conflicts of Interest: All authors have completed the ICMJE uniform disclosure form (available at http://dx.doi. org/10.21037/jtd-19-3321). The series "Radiotherapy for Brain Metastases from Lung Cancer" was commissioned by the editorial office without any funding or sponsorship. The authors have no other conflicts of interest to declare.

Ethical Statement: The authors are accountable for all aspects of the work in ensuring that questions related to the accuracy or integrity of any part of the work are appropriately investigated and resolved.

Open Access Statement: This is an Open Access article distributed in accordance with the Creative Commons Attribution-NonCommercial-NoDerivs 4.0 International License (CC BY-NC-ND 4.0), which permits the noncommercial replication and distribution of the article with the strict proviso that no changes or edits are made and the original work is properly cited (including links to both the formal publication through the relevant DOI and the license). See: https://creativecommons.org/licenses/by-nc-nd/4.0/.

\section{References}

1. Ferlay J, Colombet M, Soerjomataram I, et al. Cancer incidence and mortality patterns in Europe: estimates for 40 countries and 25 major cancers in 2018. Eur J Cancer 2018;103:356-87.

2. Ferlay J, Steliarova-Foucher E, Lortet-Tieulent J, et al. Cancer incidence and mortality patterns in Europe: estimates for 40 countries in 2012. Eur J Cancer 2013;49:1374-403.

3. Nayak L, Lee EQ, Wen PY. Epidemiology of brain metastases. Curr Oncol Rep 2012;14:48-54.

4. Davis FG, Dolecek TA, McCarthy BJ, et al. Toward determining the lifetime occurrence of metastatic brain tumors estimated from 2007 United States cancer incidence data. Neuro-Oncol 2012;14:1171-7.

5. Dawe DE, Greenspoon JN, Ellis PM. Brain metastases in non-small-cell lung cancer. Clin Lung Cancer 
2014;15:249-57.

6. Sze G, Milano E, Johnson C, et al. Detection of brain metastases: comparison of contrast-enhanced MR with unenhanced MR and enhanced CT. AJNR Am J Neuroradiol 1990;11:785-91.

7. Franchino F, Ruda R, Soffietti R. Mechanisms and therapy for cancer metastasis to the brain. Front Oncol 2018;8:161.

8. Schellinger PD, Meinck HM, Thron A. Diagnostic accuracy of MRI compared to CCT in patients with brain metastases. J Neurooncol 1999;44:275-81.

9. Server A, Josefsen R, Kulle B, et al. Proton magnetic resonance spectroscopy in the distinction of high-grade cerebral gliomas from single metastatic brain tumors. Acta Radiol 2010;51:316-25.

10. Churilla TM, Weiss SE. Emerging trends in the management of brain metastases from non-small cell lung cancer. Curr Oncol Rep 2018;20:54.

11. Sperduto PW, Kased N, Roberge D, et al. Summary report on the graded prognostic assessment: an accurate and facile diagnosis-specific tool to estimate survival for patients with brain metastases. J Clin Oncol 2012;30:419-25.

12. Paixao JV, Silva MLG, Chen MJ, et al. The shifting landscape of overall survival in patients with brain metastases in a 10-year timeframe. Int J Radiat Oncol Biol Phys 2017;99:E99.

13. Sperduto PW, Yang TJ, Beal K, et al. The effect of gene alterations and tyrosine kinase inhibition on survival and cause of death in patients with adenocarcinoma of the lung and brain metastases. Int J Radiat Oncol Biol Phys 2016;96:406-13.

14. Patchell RA, Tibbs PA, Walsh JW, et al. A randomized trial of surgery in the treatment of single metastases to the brain. N Engl J Med 1990;322:494-500.

15. Andrews DW, Scott CB, Sperduto PW, et al. Whole brain radiation therapy with or without stereotactic radiosurgery boost for patients with one to three brain metastases: phase III results the RTOG 9508 randomied trial. Lancet 2004;363:1665-72.

16. Mulvenna P, Nankivell M, Barton R, et al.

Dexamethasone and supportive care with or without whole brain radiotherapy in treating patients with nonsmall cell lung cancer with brain metastases unsuitable for resection or stereotactic radiotherapy (QUARTZ): results from a phase 3 , non-inferiority, randomised trial. Lancet 2016;388:2004-14.

17. Kondziolka D, Patel A, Lunsford LD, at al. Stereotactic radiosurgery plus whole brain radiotherapy versus radiotherapy alone for patients with multiple brain metastases. Int J Radiat Oncol Biol Phys 1999;45:427-34.

18. Sperduto PW, Shanley R, Luo X, et al. Secondary analysis of RTOG 9508, a phase 3 randomized trial of wholebrain radiation therapy versus WBRT plus stereotactic radiosurgery in patients with 1-3 brain metastases; poststratified by the graded prognostic assessment (GPA). Int J Radiat Oncol Biol Phys 2014;90:526-31.

19. Aoyama H, Shirato H, Tago M, et al. Stereotactic radiosurgery plus whole-brain radiation therapy vs stereotactic radiosurgery alone for treatment of brain metastases: a randomized controlled trial. JAMA 2006;295:2483-91.

20. DeAngelis LM, Delattre JY, Posner JB. Radiation-induced dementia in patients cured of brain metastases. Neurology 1989;39:789-96.

21. Monaco EA 3rd, Faraji AH, Berkowitz O, et al. Leukoencephalopathy after whole-brain radiation therapy plus radiosurgery versus radiosurgery alone for metastatic lung cancer. Cancer 2013;119:226-32.

22. Sun A, Bae K, Gore EM, et al. Phase III trial of prophylactic cranial irradiation compared with observation in patients with locally advanced non-small-cell lung cancer: neurocognitive and quality-of-life analysis. J Clin Oncol 2011;29:279-86.

23. Chang EL, Wefel JS, Hess KR, et al. Neurocognition in patients with brain metastases treated with radiosurgery or radiosurgery plus whole-brain irradiation: a randomised controlled trial. Lancet Oncol 2009;10:1037-44.

24. Brown PD, Asher AL, Ballman KV, et al. NCCTG N0574 (Alliance): A phase III randomized trial of whole brain radiation therapy (WBRT) in addition to radiosurgery (SRS) in patients with 1 to 3 brain metastases. J Clin Oncol 2015;33:abstr LBA4.

25. Kocher M, Soffietti R, Abacioglu U, et al. Adjuvant wholebrain radiotherapy versus observation after radiosurgery or surgical resection of one to three cerebral metastases: results of the EORTC 22952-26001 study. J Clin Oncol 2011;29:134-41.

26. Qin H, Wang C, Jiang Y, et al. Patients with single brain metastasis from non-small cell lung cancer equally benefit from stereotactic radiosurgery and surgery: a systematic review. Med Sci Monit 2015;21:144-52.

27. Brown PD, Ballman KV, Cerhan JH, et al. Postoperative stereotactic radiosurgery compared with whole brain radiotherapy for resected metastatic brain disease (NCCTG N107C/CEC-3): a multicentre, randomized, controlled, phase 3 trial. Lancet Oncol 2017;18:1049-60.

28. Yamamoto M, Serizawa T, Shuto T, et al. Stereotactic 
radiosurgery for patients with multiple brain metastases (JLGK0901): a multi-institutional prospective observational study. Lancet Oncol 2014;15:387-95.

29. Serizawa T, Yamamoto M, Higuchi Y, et al. Local tumor progression treated with Gamma Knife radiosurgery: differences between patients with 2-4 versus 5-10 brain metastases based on an update of a multi-institutional prospective observational study (JLGK0901). J Neurosurg 2019;132:1480-9.

30. ClinicalTrials.gov. Whole brain radiation versus stereotactic radiation (SRS) in patients with 5-20 brain metastases: a phase III, randomized clinical trial. Available online: https://clinicaltrials.gov/ct2/show/NCT03075072

31. ClinicalTrials.gov. Stereotactic radiosurgery compared with hippocampal-avoidant whole brain radiotherapy (HA-WBRT) plus memantine for 5-15 brain metastases.

Cite this article as: de Azevedo Rosas F, Favareto SL, Castro DG. Local ablative therapy of brain metastasis from non-small cell lung cancer: benefits and limitations. J Thorac Dis 2021;13(5):3289-3294. doi: 10.21037/jtd-19-3321
Available online: https://www.clinicaltrials.gov/ct2/show/ NCT03550391

32. Lester-Coll NH, Dosoretz AP, Magnuson WJ, et al. Costeffectiveness of stereotactic radiosurgery versus wholebrain radiation therapy for up to 10 brain metastases. J Neurosurg 2016;125:18-25.

33. Aoyama H, Tago M, Shirato H, et al. Stereotactic radiosurgery with or without whole-brain radiotherapy for brain metastases: a secondary analysis of the JROSG 99-1 randomized clinical trial. JAMA Oncol 2015;1:457-64.

34. Churilla TM, Ballman KV, Brown PD, et al. Stereotactic radiosurgery with or without whole-brain radiotherapy for limited brain metastases: secondary analysis of the North Central Cancer Treatment Group N0574 (Alliance) randomized clinical trial. Int J Radiat Oncol Biol Phys 2017;99:1173-8. 